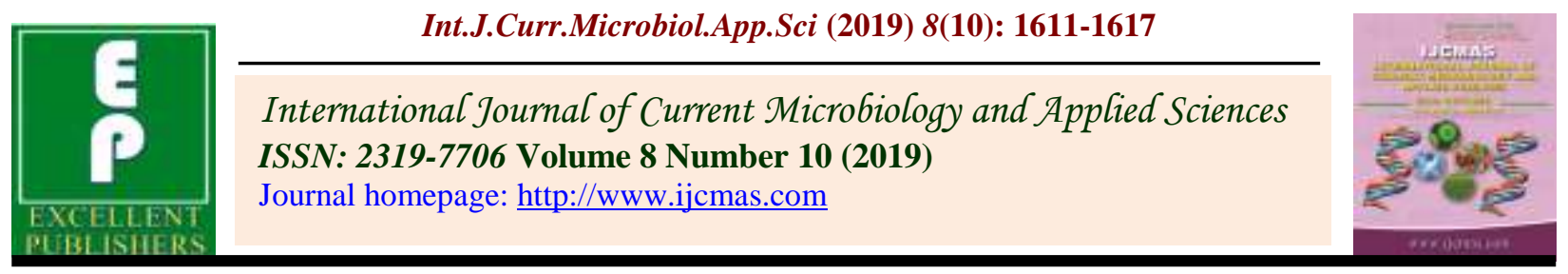

Original Research Article

https://doi.org/10.20546/ijcmas.2019.810.188

\title{
Assessment of Coriander (Coriandrum sativum L.) Genotypes for Fresh and Dry Biomass Yield under Transitional Tract of Karnataka, India
}

\author{
T. Chethan* \\ Department of Horticulture, ICAR - Krishi Vigyan Kendra, Raddewadgi (Kalaburgi II), \\ Jewargi, Kalaburgi, Karnataka-585310, India
}

Keywords

Coriandrum

sativum, Dry yield,

Fresh biomass yield, Transitional tract, Assessment

Article Info

Accepted:

12 September 2019

Available Online:

10 October 2019

\section{A B S T R A C T}

In the present study, mean performance of genotypes, based on growth and yield attributing traits of genotypes viz., DCC 5 (16.56 g), DCC 38 (16.56 g) and DCC $32(16.35 \mathrm{~g})$, followed by DCC 28 (14.69 g), DCC 35 (14.26 g), DCC 21 (13.38 g) and DCC 23 (12.21g) were identified as high yielding leafy types. Whereas, DCC 5 (2.94 g), DCC 38 (2.86 g), DCC 23 (2.85 g), DCC 35 (2.64 g), DCC 39 (2.52 g) and DCC 21 (2.42 g) recorded the maximum dry herbage yield during the whole season. These genotypes can be used successfully for further breeding programmes.

\section{Introduction}

As an annual herbaceous crop, and belonging to the Apiaceae (Umbellifera) family, coriander (Coriandrum sativum L.) is known to be native plant of Mediterranean region, Western Europe and Asia (Moniruzzaman et al., 2014 and Meena et al., 2014). It is an indispensible spice widely used as condiment throughout the world. It is mainly grown for its aromatic and fragrant seed which is botanically a cremocarpic fruit.

The fresh green stems, leaves and fruits of coriander have a pleasant aromatic odour. Green leaves of coriander are also used for culinary purposes. Dry fruits are extensively used in preparation of curry powder, pickling spices, sauces and seasonings. Good quality oleoresin can be extracted from coriander seed which is used for flavouring beverages, sweets, pickles, sausages, snacks, etc. Coriander oil has high germicidal activity and can be used as fungicide (Krishna, 1999).

In India, this crop occupies an area of 663.0 thousand hectare with the production of 609.0 thousand MT and productivity is $0.91 \mathrm{MT}$ per hectare (Anon., 2017). A germplasm collection with good variability for the desirable characters is the basic requirement of any crop improvement programme (Singhania et al., 2006). Yield and quality characters of genotype are commonly under effect of 
genetic makeup, environmental condition and agronomical practices (Gharıb et al., 2008 and Hadian et al., 2010). The aim of this study was to determine the performance and stability of coriander genotypes for fresh and dry biomass yield.

\section{Materials and Methods}

The present investigation carried out at Horticulture Research Station, Devihosur (Haveri). It comes under Transitional Zone of Karnataka state at $14^{0} 47^{\prime}$ Northern latitude, $75^{0} 21^{\prime}$ East longitudes and at an altitude of 563 meter above mean sea level. The details of source of genotypes are presented in Table 1.

Sixty one genotypes are evaluated in Randomized complete block design (RCBD) with two replications. Each genotype was raised in flat beds of $2.0 \times 1.5 \mathrm{~m}$ in size and seeds were sown with row to row distance of $15 \mathrm{~cm}$ and plant to plant distance of $15 \mathrm{~cm}$. The recommended agronomic and plant protection measures were adopted in raising good crop.

Five randomly selected plants in each genotype in each replication were tagged for recording observations on plant characters and the mean values were subjected to statistical scrutiny. Five plants in each genotype in each replication were selected randomly and tagged for recording observations for vegetative biomass yield. The mean values were used for statistical analysis. The following observations were recorded in the selected leafy type plants viz., fresh biomass yield and dry herbage yield. Fresh biomass yield was calculated as the plants were uprooted at 45th day after sowing and fresh weight of biomass along with root was taken as fresh biomass yield and expressed as gram per plant, whereas dry herbage yield calculated as plant samples were first sun dried and then kept in a solar tunnel drier at $500-600 \mathrm{C}$ for complete drying. The dry weight of whole plant sample was measured using an electronic balance and expressed in gram per plant.

\section{Fresh biomass yield}

Five separate test plants in each plot were uprooted at 45 days after sowing and weighed immediately the average weight was expressed in gram.

\section{Dry biomass yield}

The same five test plants (fresh weight of plant) were dried in solar tunnel drier at $45^{\circ}$ $50^{\circ} \mathrm{C}$ until they attained constant weight and recorded the dry weight of the plant using electric balance and expressed in gram using electric weighing machine.

\section{Results and Discussion}

During the crop season, the highest fresh biomass yield was recorded by the genotype DCC 5 (16.56 g), DCC 38 (16.56 g) and DCC $32(16.35 \mathrm{~g})$, followed by DCC $28(14.69 \mathrm{~g})$, DCC 35 (14.26 g), DCC 21 (13.38 g) and DCC $23(12.21 \mathrm{~g})$. The lowest yield was recorded by the genotype DCC 58 (6.58 g). Among the sixty one genotypes studied, twenty-nine genotypes exceeded the general mean of $10.44 \mathrm{~g}$ and thirty-five genotypes recorded the lowest than the grand mean value (Table 2).

In genotypes with increased auxin, the plants are able to absorb nutrients and translocate the nutrients to the apical bud, which leads to the conclusion that auxin acts on some protoplasmic system leading to altered arrangement of cell wall components and hence, greater extensibility leading to increased plant growth (Latha et al., 1995). The genotypes viz., DCC 5, DCC 38, DCC 32, DCC 28, DCC 35, DCC 21 and DCC 23 were 
outstanding in their growth characters, which explain for better adaptability of the genotypes under transitional zone than other genotypes. This probably attributes to the optimum or higher synthesis of carbohydrates due to increased photosynthetic efficiency resulting in better partitioning in reserved food.

This is in concordance with the works of Arif (2014), Palanikumar et al., (2012), Indiresh et al., (1990) and Rajgopalan et al., (1996) in coriander, Venkatesha (1994), Vijayalatha (2002) and Arunkumar (2003) in turmeric.

\section{Dry herbage yield/plant}

During the whole season, DCC 5and DCC 38 recorded the highest yield DCC $5(2.94 \mathrm{~g})$ and DCC 38 (2.86 g), followed by DCC 23 (2.85 g), DCC 35 (2.64 g), DCC 39 (2.52 g) and DCC $21(2.42 \mathrm{~g})$. The lowest yield was recorded by the genotype DCC 60 (1.38 g). Among sixty one genotypes studied, twenty two genotypes exceeded the general mean of
$1.97 \mathrm{~g}$ (Table 2). The highest yield of fresh and dry biomass yield was shown by the genotypes viz., DCC 5, DCC 38, DCC 32 and DCC 35.

This may be due to the suitability of soil and environmental conditions to the particular genotypes. The present findings are in conformity with the earlier results of Mohideen (1978), Dhanasekar (1997), Ann Riya (2001), Gayathri (2004), Palanikumar et al., (2012) and Arif (2014) in coriander. The genotypes DCC 5, DCC 38, DCC 32and DCC 35 were high yielding as a result of vigorous growth.

The present study revealed that, DCC 5, DCC 38, DCC 32, DCC 28, DCC 35, DCC 21 and DCC 23 genotypes were recorded maximum fresh herbage yield and DCC 5, DCC 38, DCC 32 and DCC 35 genotypes were recorded the maximum dry herbage yield during the season. The genotypes can be used for further breeding assessment.

Table.1 Name of genotypes and source of genotypes of coriander

\begin{tabular}{|c|c|c|}
\hline Treatments & Name of Variety & Source \\
\hline $\mathbf{T}_{\mathbf{1}}$ & Devihosuru Coriander Collection (DCC) -1 & H.R.S., Devihosuru (Haveri) \\
\hline $\mathbf{T}_{\mathbf{2}}$ & DCC 2 & H.R.S., Devihosuru (Haveri) \\
\hline $\mathbf{T}_{\mathbf{3}}$ & DCC 3 & H.R.S., Devihosuru (Haveri) \\
\hline $\mathbf{T}_{\mathbf{4}}$ & DCC 4 & H.R.S., Devihosuru (Haveri) \\
\hline $\mathbf{T}_{\mathbf{5}}$ & DCC 5 & H.R.S., Devihosuru (Haveri) \\
\hline $\mathbf{T}_{\mathbf{6}}$ & DCC 6 & H.R.S., Devihosuru (Haveri) \\
\hline $\mathbf{T}_{\mathbf{7}}$ & DCC 7 & H.R.S., Devihosuru (Haveri) \\
\hline $\mathbf{T}_{\mathbf{8}}$ & DCC 8 & H.R.S., Devihosuru (Haveri) \\
\hline $\mathbf{T}_{\mathbf{9}}$ & DCC 9 & H.R.S., Devihosuru (Haveri) \\
\hline $\mathbf{T}_{\mathbf{1 0}}$ & DCC 10 & H.R.S., Devihosuru (Haveri) \\
\hline $\mathbf{T}_{\mathbf{1 1}}$ & DCC 11 & H.R.S., Devihosuru (Haveri) \\
\hline $\mathbf{T}_{\mathbf{1 2}}$ & DCC 12 & H.R.S., Devihosuru (Haveri) \\
\hline $\mathbf{T}_{\mathbf{1 3}}$ & DCC 13 & H.R.S., Devihosuru (Haveri) \\
\hline $\mathbf{T}_{\mathbf{1 4}}$ & DCC 14 & H.R.S., Devihosuru (Haveri) \\
\hline $\mathbf{T}_{\mathbf{1 5}}$ & DCC 15 & H.R.S., Devihosuru (Haveri) \\
\hline $\mathbf{T}_{\mathbf{1 6}}$ & DCC 16 & H.R.S., Devihosuru (Haveri) \\
\hline $\mathbf{T}_{\mathbf{1 7}}$ & DCC 17 & H.R.S., Devihosuru (Haveri) \\
\hline $\mathbf{T}_{\mathbf{1 8}}$ & DCC 18 & H.R.S., Devihosuru (Haveri) \\
\hline $\mathbf{T}_{\mathbf{1 9}}$ & DCC 19 & H.R.S., Devihosuru (Haveri) \\
\hline
\end{tabular}




\begin{tabular}{|l|l|l|}
\hline $\mathbf{T}_{\mathbf{2 0}}$ & DCC 20 & H.R.S., Devihosuru (Haveri) \\
\hline $\mathbf{T}_{21}$ & DCC 21 & H.R.S., Devihosuru (Haveri) \\
\hline $\mathbf{T}_{22}$ & DCC 22 & H.R.S., Devihosuru (Haveri) \\
\hline $\mathbf{T}_{23}$ & DCC 23 & H.R.S., Devihosuru (Haveri) \\
\hline $\mathbf{T}_{24}$ & DCC 24 & H.R.S., Devihosuru (Haveri) \\
\hline $\mathbf{T}_{25}$ & DCC 25 & H.R.S., Devihosuru (Haveri) \\
\hline $\mathbf{T}_{26}$ & DCC 26 & H.R.S., Devihosuru (Haveri) \\
\hline $\mathbf{T}_{27}$ & DCC 27 & H.R.S., Devihosuru (Haveri) \\
\hline $\mathbf{T}_{28}$ & DCC 28 & H.R.S., Devihosuru (Haveri) \\
\hline $\mathbf{T}_{29}$ & DCC 29 & H.R.S., Devihosuru (Haveri) \\
\hline $\mathbf{T}_{30}$ & DCC 30 & H.R.S., Devihosuru (Haveri) \\
\hline $\mathbf{T}_{31}$ & DCC 31 & H.R.S., Devihosuru (Haveri) \\
\hline
\end{tabular}

\begin{tabular}{|c|c|c|}
\hline Treatments & Name of Variety & Source \\
\hline $\mathrm{T}_{32}$ & DCC 32 & H.R.S., Devihosuru (Haveri) \\
\hline $\mathbf{T}_{33}$ & DCC 33 & H.R.S., Devihosuru (Haveri) \\
\hline $\mathbf{T}_{34}$ & DCC 34 & H.R.S., Devihosuru (Haveri) \\
\hline $\mathbf{T}_{35}$ & DCC 35 & H.R.S., Devihosuru (Haveri) \\
\hline $\mathbf{T}_{36}$ & DCC 36 & H.R.S., Devihosuru (Haveri) \\
\hline $\mathbf{T}_{37}$ & DCC 37 & H.R.S., Devihosuru (Haveri) \\
\hline $\mathbf{T}_{38}$ & DCC 38 & H.R.S., Devihosuru (Haveri) \\
\hline$T_{39}$ & DCC 39 & H.R.S., Devihosuru (Haveri) \\
\hline $\mathbf{T}_{40}$ & DCC 40 & H.R.S., Devihosuru (Haveri) \\
\hline $\mathbf{T}_{41}$ & DCC 41 & H.R.S., Devihosuru (Haveri) \\
\hline$T_{42}$ & DCC 42 & H.R.S., Devihosuru (Haveri) \\
\hline$T_{43}$ & DCC 43 & H.R.S., Devihosuru (Haveri) \\
\hline $\mathbf{T}_{44}$ & DCC 44 & H.R.S., Devihosuru (Haveri) \\
\hline$T_{45}$ & DCC 45 & H.R.S., Devihosuru (Haveri) \\
\hline$T_{46}$ & DCC 46 & H.R.S., Devihosuru (Haveri) \\
\hline $\mathbf{T}_{47}$ & DCC 47 & H.R.S., Devihosuru (Haveri) \\
\hline$T_{48}$ & DCC 48 & H.R.S., Devihosuru (Haveri) \\
\hline$T_{49}$ & DCC 49 & H.R.S., Devihosuru (Haveri) \\
\hline$T_{50}$ & DCC 51 & H.R.S., Devihosuru (Haveri) \\
\hline $\mathbf{T}_{51}$ & DCC 52 & H.R.S., Devihosuru (Haveri) \\
\hline$T_{52}$ & DCC 53 & H.R.S., Devihosuru (Haveri) \\
\hline$T_{53}$ & DCC 54 & H.R.S., Devihosuru (Haveri) \\
\hline $\mathbf{T}_{54}$ & DCC 55 & H.R.S., Devihosuru (Haveri) \\
\hline $\mathbf{T}_{55}$ & DCC 56 & H.R.S., Devihosuru (Haveri) \\
\hline$T_{56}$ & DCC 57 & H.R.S., Devihosuru (Haveri) \\
\hline $\mathbf{T}_{57}$ & DCC 58 & H.R.S., Devihosuru (Haveri) \\
\hline$T_{58}$ & DCC 59 & H.R.S., Devihosuru (Haveri) \\
\hline $\mathbf{T}_{59}$ & DCC 60 & H.R.S., Devihosuru (Haveri) \\
\hline$T_{60}$ & DWR 1 & H.R.S., Devihosuru (Haveri) \\
\hline$T_{61}$ & Ajjampura local & H.R.S., Devihosuru (Haveri) \\
\hline
\end{tabular}


Table.2 Mean performance of coriander genotypes for fresh biomass and dry herbage yield $\mathrm{g} / \mathrm{plant}$

\begin{tabular}{|c|c|c|c|}
\hline Sl. No. & Genotypes & Fresh weight (g/plant) & Dry weight (g/plant) \\
\hline $\mathbf{1}$ & DCC 1 & 8.12 & 1.48 \\
\hline $\mathbf{2}$ & DCC 2 & 8.17 & 1.53 \\
\hline $\mathbf{3}$ & DCC 3 & 12.46 & 2.31 \\
\hline $\mathbf{4}$ & DCC 4 & 9.09 & 1.65 \\
\hline $\mathbf{5}$ & DCC 5 & 16.56 & 2.86 \\
\hline $\mathbf{6}$ & DCC 6 & 7.20 & 2.12 \\
\hline $\mathbf{7}$ & DCC 7 & 8.77 & 1.91 \\
\hline $\mathbf{8}$ & DCC 8 & 10.41 & 1.84 \\
\hline $\mathbf{9}$ & DCC 9 & 8.88 & 1.77 \\
\hline $\mathbf{1 0}$ & DCC 10 & 9.61 & 1.89 \\
\hline $\mathbf{1 1}$ & DCC 11 & 11.04 & 2.24 \\
\hline $\mathbf{1 2}$ & DCC 12 & 10.13 & 1.78 \\
\hline $\mathbf{1 3}$ & DCC 13 & 8.69 & 1.63 \\
\hline $\mathbf{1 4}$ & DCC 14 & 6.61 & 1.48 \\
\hline $\mathbf{1 5}$ & DCC 15 & 8.46 & 1.45 \\
\hline $\mathbf{1 6}$ & DCC 16 & 11.43 & 2.17 \\
\hline $\mathbf{1 7}$ & DCC 17 & 8.33 & 2.02 \\
\hline $\mathbf{1 8}$ & DCC 18 & 10.46 & 1.96 \\
\hline $\mathbf{1 9}$ & DCC 19 & 8.20 & 1.46 \\
\hline $\mathbf{2 0}$ & DCC 20 & 11.98 & 1.91 \\
\hline $\mathbf{2 1}$ & DCC 21 & 13.38 & 2.42 \\
\hline $\mathbf{2 2}$ & DCC 22 & 9.85 & 1.80 \\
\hline $\mathbf{2 3}$ & DCC 23 & 12.21 & 2.52 \\
\hline $\mathbf{2 4}$ & DCC 24 & 10.22 & 2.21 \\
\hline $\mathbf{2 5}$ & DCC 25 & 12.85 & 2.14 \\
\hline $\mathbf{2 6}$ & DCC 26 & 11.05 & 2.07 \\
\hline $\mathbf{2 7}$ & DCC 27 & 7.41 & 1.56 \\
\hline $\mathbf{2 8}$ & DCC 28 & 14.69 & 1.92 \\
\hline $\mathbf{2 9}$ & DCC 29 & 9.16 & 2.06 \\
\hline $\mathbf{3 0}$ & DCC 30 & 10.33 & 1.66 \\
\hline & & & \\
\hline & & & \\
\hline
\end{tabular}

Contin...

\begin{tabular}{|c|c|c|c|}
\hline Sl. No. & Genotypes & Fresh weight (g/plant) & Dry weight (g/plant) \\
\hline $\mathbf{3 1}$ & DCC 31 & 9.52 & 1.75 \\
\hline $\mathbf{3 2}$ & DCC 32 & 16.35 & 1.84 \\
\hline $\mathbf{3 3}$ & DCC 33 & 12.04 & 1.75 \\
\hline $\mathbf{3 4}$ & DCC 34 & 11.00 & 2.59 \\
\hline $\mathbf{3 5}$ & DCC 35 & 14.26 & 2.64 \\
\hline $\mathbf{3 6}$ & DCC 36 & 12.36 & 1.95 \\
\hline $\mathbf{3 7}$ & DCC 37 & 11.52 & 1.99 \\
\hline $\mathbf{3 8}$ & DCC 38 & 16.56 & 2.85 \\
\hline $\mathbf{3 9}$ & DCC 39 & 10.84 & 2.94 \\
\hline $\mathbf{4 0}$ & DCC 40 & 11.46 & 1.83 \\
\hline $\mathbf{4 1}$ & DCC 41 & 9.30 & 1.93 \\
\hline $\mathbf{4 2}$ & DCC 42 & 11.47 & 2.44 \\
\hline $\mathbf{4 3}$ & DCC 43 & 9.14 & 1.50 \\
\hline $\mathbf{4 4}$ & DCC 44 & 8.97 & 1.41 \\
\hline
\end{tabular}




\begin{tabular}{|l|c|c|c|}
\hline $\mathbf{4 5}$ & DCC 45 & 11.43 & 2.15 \\
\hline $\mathbf{4 6}$ & DCC 46 & 12.10 & 1.86 \\
\hline $\mathbf{4 7}$ & DCC 47 & 7.31 & 1.51 \\
\hline $\mathbf{4 8}$ & DCC 48 & 10.66 & 2.03 \\
\hline $\mathbf{4 9}$ & DCC 49 & 11.58 & 2.05 \\
\hline $\mathbf{5 0}$ & DCC 51 & 7.87 & 1.65 \\
\hline $\mathbf{5 1}$ & DCC 52 & 10.53 & 1.70 \\
\hline $\mathbf{5 2}$ & DCC 53 & 10.44 & 1.92 \\
\hline $\mathbf{5 3}$ & DCC 54 & 8.25 & 1.55 \\
\hline $\mathbf{5 4}$ & DCC 55 & 9.75 & 2.12 \\
\hline $\mathbf{5 5}$ & DCC 56 & 10.94 & 1.91 \\
\hline $\mathbf{5 6}$ & DCC 57 & 11.32 & 2.36 \\
\hline $\mathbf{5 7}$ & DCC 58 & 6.58 & 2.20 \\
\hline $\mathbf{5 8}$ & DCC 59 & 12.30 & 2.24 \\
\hline $\mathbf{5 9}$ & DCC 60 & 7.38 & 1.38 \\
\hline $\mathbf{6 0}$ & DWR-1 & 8.97 & 2.54 \\
\hline $\mathbf{6 1}$ & Ajjampura Local & 9.15 & 1.75 \\
\hline & Mean & $\mathbf{1 0 . 4 4}$ & $\mathbf{1 . 9 7}$ \\
\hline & S.Em \pm & 1.57 & 0.34 \\
\hline & C.D. at 5\% & 4.45 & 0.97 \\
\hline & C.V. & 21.3 & 21.60 \\
\hline
\end{tabular}

\section{References}

Anonymous, 2017, Horticulture statistics at a glance 2017. National horticulture board, 25(4):7-15.

Arif, A., 2014, Evaluation of coriander (Coriandrum sativum L.)genotypes for fresh and dry biomass yield under hill zoneof Karnataka. Int. J. Agri. Sci., 10(2): 747-750.

Ann Riya, 2001, Evaluation of genotypes and effect of nutrition on clipping in coriander (Coriandrum sativum L.). M.Sc. (Hort.) Thesis, Tamil Nadu Agricultural University, Coimbatore, T.N. (INDIA).

Arunkumar, R., 2003, Evaluation of turmeric accession for yield, quality and good shoot borer resistance. Ph.D. (Hort.) Thesis, Tamil Nadu Agricultural University, Coimbatore, T.N. (INDIA).

Dhanasekar, D., 1997, Screening of coriander (Coriandrum sativum L.) genotypes for green yield and quality. M.Sc. (Hort.) Thesis, Tamil Nadu Agricultural University, Coimbatore,
T.N. (INDIA).

Gayathri, S., 2004, Evaluation of coriander (Coriandrum sativum L.) genotypes for high leaf and grain yield. M.Sc. (Hort.) Thesis, Tamil Nadu Agricultural University, Coimbatore, T.N. (INDIA).

Gharib, F.A., Lobna, A., Moussa, L.A. and Massoud, O.N., 2008. Effect of Compost and Bio-fertilizers on Growth, Yield and Essential Oil of Sweet Marjoram (Majorana hortensis) Plant. Int. J. Agri. Biol., 10(4): 381387.

Hadian, J., Ebrahimi, S.N. and Salehi, P., 2010, Variability of morphological and phytochemical characteristics among Satureja hortensis L. accessions of Iran. Industrial Crops and Products, 32(1): 62-69.

Indiresh, K.M., Uthaiah, B.C., Reddy, M.J. and Rao, K.B., 1990, Morphological rhizome and yield characters of different turmeric varieties in coastal Karnataka. Mysore J. Agric. Sci., 24: 484-490. 
Krishna, D. A., 1999, Spice: Some known and unknown facts. Sci. and Cult., 65: 22028.

Latha, P., Giridharan, M. P. and Naik, B. J., 1995, Performance of turmeric (Curcuma longa L.) cultivars in open and partially shaded condition under coconut. J. Spices \& Aromatic Crops, 2: 139-144.

Meena, Y.K., Jadhao, B.J. and Kale, V.S., 2014, Genetic analysis of agronomic traits in coriander. Sabrao J. Breed. Gen., 46(2): 265-273.

Mohideen, D.C., 1978,Studies on varieability, correlation and path analysis in (Amaranthus gangeticus L.). M.Sc. (Ag.) Thesis, Tamil Nadu Agricultural University, Coimbatore, T.N. (INDIA). Moniruzzaman, M., Rahman, M.M., Hossain, M.M., Karim, A.J.M.S. and Khaliq, Q.A., 2014, Response of Coriander (Coriandrum sativum L.) foliage to different rates and methods of nitrogen application. Bangladesh J. Agril. Res., 39(2): 359-371.

Palanikumar, M., Rajamani, K. and Muthaiah, A.R., 2012, Correlation studies in coriander (Coriandrum sativum L.) genotypes for fresh biomass yield and oil content under different seasons. Crop Res., 44(1\&2): 217-221.

Rajgopalan, A., Azhakiyamanavalan, R. S. and Khader, M. D. A., 1996, Evaluation of coriander cultivars for yield and quality. Indian Cocoa, Arecanut \& Spices J., 20 : 13-14.

Singhania, D.L., Singh, D. and Raje.R. S., 2006, Advances in Spices and Achievements of Spices Research in India since Independence.Agrobios, 678-695.

Venkatesh, J., 1994,Studies on evaluation of promising cultivars and nutrient requirement of turmeric (Curcuma domestica Vel.). Ph.D. (Hort.) Thesis, University of Agricultural Science, Bangalore, KARNATAKA (INDIA).

Vijaylatha, K. R., 2002, Genetic divergence, multivariate analysis and molecular markers in turmeric (Curcum longa L.). Ph.D. (Hort.) Thesis, Tamil Nadu Agricultural University, Coimbatore, T.N. (INDIA).

\section{How to cite this article:}

Chethan, T. 2019. Assessment of Coriander (Coriandrum sativum L.) Genotypes for Fresh and Dry Biomass Yield under Transitional Tract of Karnataka. Int.J.Curr.Microbiol.App.Sci. 8(10): 1611-1617. doi: https://doi.org/10.20546/ijcmas.2019.810.188 